\title{
The Effect of COVID-19 on the Reasons to Utilize Dental Emergency Services, Do We Need a New Dental Care Model?
}

\author{
Omar Bamedhaf ${ }^{1}$ and Shiamaa Shihab Almashhadani ${ }^{2, *}$ \\ ${ }^{1}$ Specialist Prosthodontist, Dental Services Department, Dubai Health Authority, United Arab Emirates \\ ${ }^{2}$ Senior Specialist registrar, Dental Public Health Specialist, Dental Services Department, Dubai Health Authority, United Arab Emirates
}

*Corresponding author: Shiamaa Shihab Almashhadani, Senior Specialist registrar, Dental Public Health Specialist, Dental Services Department, Dubai Health Authority, United Arab Emirates, E-mail: ssalmashhadani@dha.gov.ae

Received: 19 Feb, 2021 | Accepted: 09 Mar, 2021 | Published: 19 Mar, 2021

Citation: Bamedhaf O, Almashhadani SS (2021) The Effect of COVID-19 on the Reasons to Utilize Dental Emergency Services, Do We Need a New Dental Care Model? Int J Dent Oral Health 7(3): dx.doi.org/10.16966/2378-7090.354

Copyright: (C2021 Bamedhaf O, et al. This is an open-access article distributed under the terms of the Creative Commons Attribution License, which permits unrestricted use, distribution, and reproduction in any medium, provided the original author and source are credited.

\section{Abstract}

Aim: To assess how COVID-19 effected the patients' utilization of dental services and how the pandemic will change dental care services.

Methods: Patients' visits during the sterilization program period during April 2020 were retrieved. Their demographics and reasons for dental visits were recorded and analyzed.

Results: There were 272 patients involved in the study. There was no significant difference between males $(50.7 \%)$ and females $(49.3 \%)$. The mean age was 40 years $( \pm 10$ [5-78]). Dental emergencies ranged from $15 \%$ of a patient complaining of pain from dental caries (15.1\%) and pulp involvement $(15.4 \%)$, with a significant number of patients $(25.4 \%)$ effected by incomplete dental treatment procedures.

Conclusion: The study has provided an insight within its limitation of emergency dental needs during a crisis or pandemic, which requires consideration to evaluate the current dental care model and recommendations for future crisis and pandemics oral health needs.

Keywords: COVID; Dental care; Aerosols; Transmission; New dental model; Emergency

\section{Introduction}

Coronavirus (COVID-19), is a new strain of coronavirus similar to SARS-CoV and the Middle East respiratory syndrome (MERS-CoV) that causes a respiratory infection ranging from the common cold to more severe diseases [1]. The virus and the disease it causes were never known before. It spread across the world starting from Wuhan city of Hubei, a province in China, in December 2019. By February 2020 the disease had spread to more than 34 countries where the World Health Organization announced that the disease was a public health emergency of international concern [2]. The source of the Novel Coronavirus (COVID-19) has not been identified; with studies suggesting the Chinese horseshoe (Rhinolophus sinicus) [3]. However, most of the cases were epidemiologically linked to the seafood and animal market in Wuhan, China, as the initial spread happened amongst the shoppers who were present there [4]. There are current studies taking place to determine the actual source of the disease [1].

\section{Mode of transmission}

COVID-19 is understood to be spreading from human to human via direct or indirect contact with infected people via infected respiratory droplets or saliva, fomites, or aerosols droplets [5]. Some of the other possible modes of transmission are being explored by the researchers, with some studies suggesting the viral spread through fecal-oral, conjunctival secretions and direct contact with contaminated surfaces or hands [6].

Of particular interest to dental care providers is the transmission of the virus by saliva. Angiotensin-Converting Enzyme-II (ACE-II) is supposed to be the receptor for SARS-CoV and SARS-CoV-2. It is the viral binding enzyme found in abundance in the epithelial cells of the oral mucosa and can be transmitted through saliva [6]. The positive viral culture in the study by To et al indicated that saliva contains live viruses and may allow transmission. Saliva may also contain viruses coming down from the nasopharynx or coming up from the lung via the airway [7].

\section{Signs and symptoms of SARS-COVID-19}

The epidemiological arguments as contact with a confirmed SARSCoV-2 infected patient or return from an "at-risk area" with SARSCoV-2 circulation are crucial to suspect a case of COVID-19 [8]. The major clinical symptoms of the SARS-CoV-2 are influenza-like which comprise fever, cough, headache, generalized myalgia, malaise, drowsiness, gastrointestinal disturbance such as vomiting and 
diarrhoea, confusion, loss of taste and smell, dyspnoea, rhinorrhoea, pneumonia and rigorous shivering [9].

\section{Precautionary measures}

Safeguards to prevent the spread of COVID-19 include sneezing and coughing etiquette, avoid touching the eyes, nose or mouth by hand without washing, avoiding direct contact (handshaking).

The UAE registered 814 cases beginning of April 2020 with 8 deaths and more than 240,000 tests done [10]. This called for immediate action from the authorities to start a sterilization campaign as an effort to contain COVID-19. Night curfew was imposed later while the country began disinfection, which later led to an all-day curfew. Schools, universities and public facilities were closed down. All health care authorities postponed elective care and only emergency cases were treated in health facilities.

\section{COVID-19 and the dental care}

Experts had theorized that the coronavirus, which resides in the saliva, throat and nasal passages of infected patients, can be propelled into the air if the patient coughs or gags, thus posing a threat to medical personnel and patients [1].

In addition, the US Centres for Disease Control had announced that the use of rotary dental and surgical instruments in dentistry, such as hand-pieces, ultrasonic scalers and air-water syringes, create a visible spray that can contain particles droplets of water, saliva and microorganisms. While surgical masks protect mucous membranes of the mouth and nose from droplet spatter, they do not provide complete protection against the inhalation of airborne and aerosolised infectious agents [11].

Therefore, due to the high risk of cross-infection between patients and dental practices, dental services were postponed during the pandemic and were limited to emergency cases with strict infection control procedures.

There were two health centres out of 13 government primary health care facilities, which were available for the emergency services during the sterilization program. Dental care facilities in the primary health care centres, Dubai health authority were all postponed.

A phone encounter triaging system was introduced to allow access to dental care with a questioning protocol and was limited to emergency cases, providing the patients with assigned slots to maintain social distancing with strict infection control procedures.

Health facilities both governmental and private providing dental services during the COVID-19 pandemic followed a dental care emergency guideline published by the health regulation department, DHA [12].

These facilities could provide emergency and urgent dental care that includes:

a) Trauma resulting in fracture or displacement of a tooth, alveolar bone, or soft tissue laceration.

b) Oro-facial swelling that compromises swallowing and/or breathing.

c) Post-extraction bleeding that is not responding to self-care measures.

d) Severe pain or trismus that is not responding to over-thecounter analgesics.

e) Non-healing mouth ulcers.
Urgent dental care is determined by the clinicians to either establish or maintain a patient's oral health. Such determinations are based on the professional diagnostic judgment of the dentist, and the standards of care that prevail in the professional community [12].

This paper will provide the main reasons patients sought to utilize dental emergencies provided during the COVID-19 crisis and recommendations for future crisis and pandemics oral health needs.

\section{Materials and Methodologies}

A retrospective analysis was done retrieving patients seeking emergency dental services from the electronic database "Epic "Salama" starting from the $1^{\text {st }}$ to the $30^{\text {th }}$ of April 2020 where the dental services were postponed due to the COVID-19 outbreak. One location was checked (Al-Barsha health center) which provided dental emergency services. The data divided into demographics of the patients and reasons of the visit for patients seeking emergency dental service through a triage phone encounter in Dubai Health Authority (DHA) during the mentioned period.

The reasons were divided into categories based on pain-related visits and others. The data was inserted in an encrypted excel sheet accessible only to the authors. Continuous variables as a mean standard deviation, categorical variables presented as $\mathrm{n}(\%)$ and analysed using chi-square with SPSS version 20.0. The significance level is set at 0.05 .

\section{Ethical Approval}

Ethics approval was obtained from Dubai Scientific Research Ethics Committee (DSREC), Dubai Health Authority, Ref: DSREC-09/2020_08 obtained on 13 SEP 2020.

\section{Results}

272 patients were seen in both clinics after phone encounter triage. There was no significant difference between males (50.7\%) and females $(49.3 \%)$. The age range from 5 years to 78 years with a mean of 40 years $( \pm 10$ [5-78]). Full demographics are shown in table 1.

Analysis of the reasons for visits identified a salient number of patients complaining of pain from pulpal involvement (15.4\%) followed by pain from dental caries $(15.1 \%)$ and fractured fillings and teeth (13.6\%) (Table 2).

The study shows a change of patient flow with peak visits during the middle of the month (Figure 1 ).

A significant number of patients (25.4\%) were effected from incomplete dental procedures that were postponed due to the crisis.

Table 1: Age and Gender Distribution of the study population.

\begin{tabular}{|l|l|c|c|}
\hline Variables & \multicolumn{1}{|c|}{ Categories } & $\mathbf{n}$ & \% \\
\hline \multirow{4}{*}{ Age } & Less than 10 Years & 31 & 11.4 \\
\cline { 2 - 4 } & $11-20$ Years & 37 & 13.6 \\
\cline { 2 - 4 } & 21-30 Years & 48 & 17.6 \\
\cline { 2 - 4 } & 31-40 Years & 64 & 23.5 \\
\cline { 2 - 4 } & 41-50 Years & 44 & 16.2 \\
\cline { 2 - 4 } & 51-60 Years & 24 & 8.8 \\
\cline { 2 - 4 } & 60 Years and Above & 24 & 8.8 \\
\hline \multirow{2}{*}{ Gender } & Male & 138 & 50.7 \\
\cline { 2 - 4 } & Female & 134 & 49.3 \\
\hline
\end{tabular}


Table 2: Distribution of the study participant according to the reason of visit (Dental Caries and related)

\begin{tabular}{|l|c|c|c|c|}
\hline \multirow{2}{*}{\multicolumn{1}{|c|}{ Reasons for the visit }} & \multicolumn{2}{c|}{ Absent } & \multicolumn{2}{c|}{ Present } \\
\cline { 2 - 5 } & $\mathrm{n}$ & $\%$ & $\mathrm{n}$ & $\%$ \\
\hline Dental pain caused by dental caries & 231 & 84.9 & 41 & 15.1 \\
\hline $\begin{array}{l}\text { Dental pain caused by irreversible pulpitis/ } \\
\text { necrotic pulp }\end{array}$ & 230 & 84.6 & 42 & 15.4 \\
\hline Presence of remaining roots & 260 & 95.6 & 12 & 4.4 \\
\hline Lost filling, broken filling or restored tooth & 235 & 86.4 & 37 & 13.6 \\
\hline Incomplete RCT & 240 & 88.2 & 32 & 11.8 \\
\hline Periapical disease (post RCT) & 252 & 92.6 & 20 & 7.4 \\
\hline
\end{tabular}

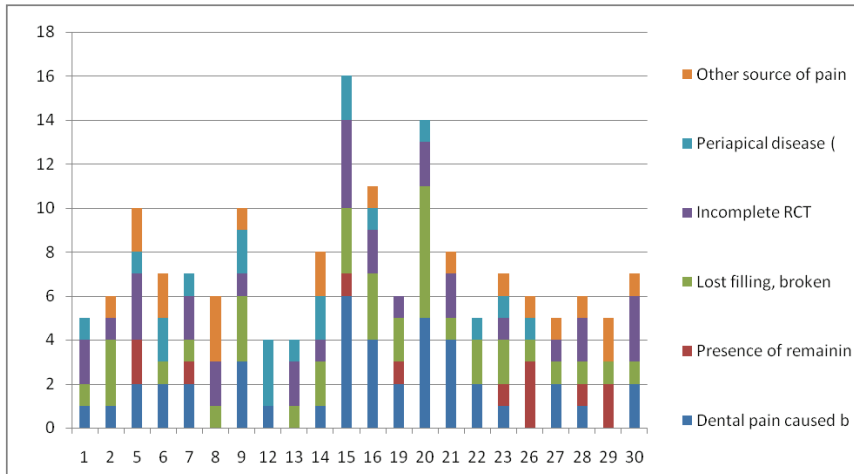

Figure 1: Association between the date and the reason for visit.

The procedures were incomplete endodontic cases, prosthodontic crown \& bridge, and orthodontic procedures (Table 3).

Patients also visited the clinic due to soft tissue involvement with suffering from pericoronitis (7.4\%), periodontal involvement (7.7\%), with only 2 patients attending the clinic due to cellulitis (Table 4).

\section{Discussion and Conclusion}

The study provides a descriptive analysis of the data in April 2020 and prominent findings of the main reasons of patients who utilized emergency dental care during this period. The current COVID situation has caused a drastic change in the pattern of delivery of dental care. This is demonstrated with 2400 calls for dental advice registered from phone encounters during April. Delayed dental care has dire consequences on patients causing multiple complications, as it has been clear with $25 \%$ of visits during the sterilization program to be due to incomplete dental care. The lack of routine dental care also reflects negatively as there is an increase in pain and discomfort leaving the patient with no option other than visiting emergency rooms in hospitals. With the COVID situation and the overload of hospitals, patients who suffer from dental and oral pain are not prioritized. Furthermore, the postponing of dental procedures creates a significant backlog of patients requiring the completion of their treatment and new urgent cases that require the attention of dental health providers during the post-COVID time [13]. Considerations should be placed to set up the dental care system to provide quality oral health care with minimum disruption of providing the service to the public.

In a report on the COVID-19 and Dentistry: Challenges and Opportunities for Providing Safe Care [14], recommendations were made to adopt an innovative dental model. The model would include
Table 3: The distribution of study participants visits according to other reasons of pain.

\begin{tabular}{|l|c|c|}
\hline \multicolumn{1}{|c|}{ Source of pain } & N & $\%$ \\
\hline Pericoronitis & 20 & 7.4 \\
\hline Orthodontic discomfort & 11 & 4.0 \\
\hline TMJ & 3 & 1.1 \\
\hline Tooth Fracture & 1 & 0.4 \\
\hline Mobile Implant & 3 & 1.1 \\
\hline Teeth Sensitivity & 1 & 0.4 \\
\hline Post Op Extraction complications & 2 & 0.7 \\
\hline Soft tissue injuries & 4 & 1.5 \\
\hline Crown and bridge & 13 & 4.8 \\
\hline Tooth mobility & 2 & 0.7 \\
\hline Others & 2 & 0.7 \\
\hline Total & 63 & 100 \\
\hline
\end{tabular}

Table 4: The association between the age and periodontal reasons for visit.

\begin{tabular}{|c|c|c|c|c|c|c|c|}
\hline \multicolumn{8}{|c|}{ Periodontal Reasons } \\
\hline & & \multicolumn{2}{|c|}{ Gingivitis } & \multicolumn{2}{|c|}{ Periodontitis } & \multicolumn{2}{|c|}{ Abscess } \\
\hline & & $\mathbf{n}$ & $\%$ & $n$ & $\%$ & $\mathbf{n}$ & $\%$ \\
\hline \multirow{7}{*}{ Age } & Less than 10 Years & 2 & 15.38 & - & - & - & - \\
\hline & $11-20$ Years & 2 & 15.38 & - & - & - & - \\
\hline & 21-30 Years & 2 & 15.38 & - & - & - & - \\
\hline & $31-40$ Years & 1 & 7.69 & 1 & 12.5 & 1 & 50 \\
\hline & 41-50 Years & 5 & 38.4 & 5 & 62.5 & - & - \\
\hline & 51-60 Years & 0 & - & 2 & 25 & & - \\
\hline & 60 Years and Above & 1 & 7.69 & 0 & & 1 & 50 \\
\hline Total & & 13 & & 8 & & 2 & \\
\hline
\end{tabular}

the use of available resources to provide a solution for future dental care during and after epidemics or other emergencies.

Giudice A, et al., [15], Watt RG [16], Spagnuolo G, et al., [17] and Meng L, et al., [4] have explored the different aspects of COVID -19 in relation to dental care. The pandemic has given the health care industry insight into the many pitfalls and gaps in health systems. Be it inequalities in health care, the health care providers' impact and affect, financial and insurance cover or environmental and industrial factors.

As a recommendation, we suggest the following framework to highlight all the main changes to be considered in the dental care industry during a post-COVID era. Furthermore, the lessons learned during this pandemic should allow us to face the future with robust and insightful plans. The framework recommends further in-depth research and solutions for three main factors; Environmental factors, Dental health provider factors and Patient factors.

\section{Environmental and dental health provider factors}

The financial impact of COVID-19 has taken its toll on the dental care industry due to the international policies to limit dental practice and cease all elective dental procedures to prevent an outbreak of the disease. Governments should consider designing subsidy and safety nets including immediate, midterm and long-term economic 
relief effects that will help during and after a pandemic [18]. Dental practices should plan to have minimum profitability on minimum sustainability. Furthermore, to support dental practices we must not forget the role of digital and technology solutions. Teledentistry has played an important role in providing novel solutions to resume dental practice during the current pandemic; hence, the need of the hour is to incorporate teledentistry into routine dental practice. It can complement the existing compromised dental system during the current pandemic. Teleconsultation, telediagnosis, tele-triage, and telemonitoring are aspects that can be explored and expanded to support the dental industry in current and post-pandemic situations [19].

Technology can also play a role in the training of dental personnel to handle situations and new procedures due to pandemics. Simulation training in infection control, PPE, use of equipment necessary to prevent the spread of disease not only protects the health care provider and the community it will strengthen the resilience and well-being of dental providers [20].

The role the dental provider has participated in during this pandemic has shown that they can be incorporated in an integrated health model of care that serves the new vision of health. A new human-centered global healthcare framework should be considered that takes into account the dynamic situation during and post a pandemic. All multidisciplinary industries and sectors of the societies should be involved [21,22].

Further to the new health vision, having the autonomy to expand the dental team to include dental hygienists, therapists and educators will have a positive impact on reducing costs, controlling emergencies and complications during and after a pandemic.

\section{Patient factors}

Spagnuolo G, et al., describes the definition of patient groups and the preferred setting of treatment for each category. In addition, setting the urgency of treatment with guidelines to understand what qualifies an emergency, urgency or a routine continuation of treatment and whether the category of "routine dental visit" will cause a complication if postponed [17].

One of the lessons learned during this pandemic is that we face a dynamic changing situation. Screening tests have evolved rapidly during the COVID-19 outbreak and there is urgency for research to substantiate serological tests with fast results. The latter will help in triaging and screening patients attending dental care establishments, support dental care for patients during the spread of disease and prevent further complications of oral health problems by providing the service without delay [15].

We must not forget that prevention and promotion are vital to support the patient in improving oral health, reduce the risk of complications and the need to seek urgent care. The invaluable results of early prevention and intervention will be reflected during pandemics and crises $[23,24]$.

In conclusion, the demands of the population for dental care whether emergency, urgent or routine care should be met to provide a high quality of life and diminish inequalities in health care. The dental health care system should be able to meet the needs and provide the

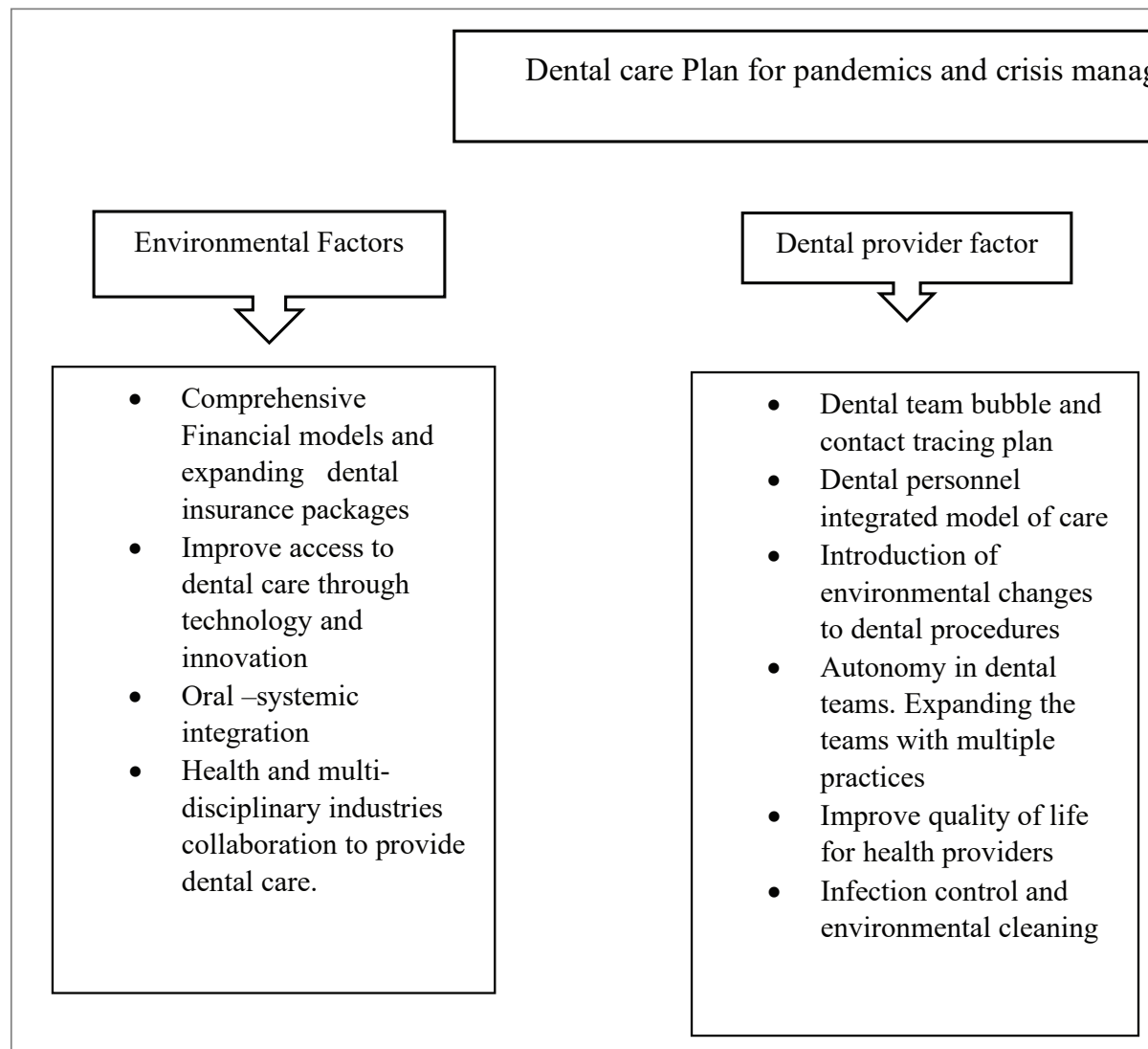

Figure 2: Dental care plan for pandemics management. 
care required for the community it serves during any circumstances. The study within its limitation due to data collected from one centre providing emergency dental services in a certain geographic area which may not represent the full population, A future research including a representative sample from the population not inclusive to only emergency dental services in the government sector is suggested to provide recommendations for a new dental care model to adapt all forms of oral health care needs during a pandemic or crisis (Figure 2).

\section{Acknowledgments}

The authors would like to thank the dental services department for providing access to dental records.

\section{Conflicts of Interest}

The authors declare no conflicts of interest.

\section{Funding}

The authors have not received funding for the study.

\section{Authors Contribution}

Both authors have contributed equally to the study.

\section{References}

1. Guo H, Zhou Y, Liu X, Tan J (2020) The impact of the COVID-19 epidemic on the utilization of emergency dental services. J Dent Sci 15: 564-567.

2. American Dental Association (2020) ADA urges dentists to heed April 30 interim postponement recommendation, maintain focus on urgent and emergency dental care only.

3. Mackenzie JS, Smith DW (2020) COVID-19: a novel zoonotic disease caused by a coronavirus from China: what we know and what we don't. Microbiol Aust 41: 45-50.

4. Meng L, Hua F, Bian Z (2020) Coronavirus disease 2019 (COVID-19): emerging and future challenges for dental and oral medicine. J Dent Res 99: 481-487.

5. Madewell ZJ, Yang Y, Longini IM, Halloran ME, Dean NE (2020) Household Transmission of SARS-CoV-2: A Systematic Review and Meta-analysis. JAMA Netw Open 3: e2031756.

6. Mukhra R, Krishan K, Kanchan T (2020) Possible modes of transmission of novel coronavirus SARS-CoV-2: A review. Acta Biomed 91: e2020036.

7. To KK, Tsang OT, Yip CC, Chan KH, Wu TC, et al. (2020) Consistent Detection of 2019 Novel Coronavirus in Saliva. Clin Infect Dis 71 : 841-843.

8. Zayet $\mathrm{S}$, Kadiane-Oussou NJ, Lepiller Q, Zahra H, Royer PY, et al. (2020) Clinical features of COVID-19 and influenza: a comparative study on Nord Franche-Comte cluster. Microbes Infect 22: 481-488.
9. Cohen PA, Hall LE, John JN, Rapoport AB (2020) The early natural history of SARS-CoV-2 infection: clinical observations from an urban, ambulatory COVID-19 Clinic. Mayo Clin Proc 95: 1124-1126.

10. Gulf News (2020) COVID-19: UAE announces 41 new coronavirus cases. UAE.

11. Bhanushali $P$, Katge F, Deshpande $S$, Chimata VK, Shetty $S$, et al. (2020) COVID-19: Changing trends and its impact on future of dentistry. Int J Dent.

12. Health Policies and Standards Department, Health Regulation Sector (2020) Guidelines for the Provision of Dental Services during COVID-19. Dubai Health Authority, Government of Dubai.

13. Grossman S, Sandhu P, Sproat C, Patel V (2020) Provision of dental services at a single institution in the UK's epicentre during the COVID-19 pandemic. Br Dent J 228: 964-970.

14. Kalenderian E, Xiao Y, Spallek H, Franklin A, Olsen G, et al. (2020) COVID-19 and Dentistry: Challenges and Opportunities for Providing Safe Care. PSNET.

15. Giudice A, Bennardo F, Antonelli A, Barone S, Fortunato L (2020) COVID-19 is a new challenge for dental practitioners: advice on patients' management from prevention of cross infections to telemedicine. Open Dent J 14: 298-304.

16. Watt RG (2020) COVID-19 is an opportunity for reform in dentistry. Lancet 396: 462.

17. Spagnuolo G, De Vito D, Rengo S, Tatullo M (2020) COVID-19 outbreak: an overview on dentistry. Int J Environ Res Public Health 17: 2094.

18. Schwendicke F, Krois J, Gomez J (2020) Impact of SARS-CoV2 (COVID-19) on dental practices: Economic analysis. J Dent 99: 103387.

19. Ghai S (2020) Teledentistry during COVID-19 pandemic. Diabetes Metab Syndr 14: 933-935.

20. Cheung VKL, So EHK, Leung ASH, Chia NH (2020) Caring for Healthcare Professionals during COVID-19 Crisis: Integrating Simulation Training with Hospital System towards Actualizing Occupational Safety, Health and Well-being. J Clin Immunol Immunother 6: 45.

21. Brown C, Harrison D, Burns H, Ziglio E (2013) Governance for health equity. WHO Regional Office for Europe.

22. Kher R, Nair AS (2020) The Case for a Global Healthcare Partnership. RIS.

23. Watt R, Fuller S (1999) Oral health promotion-opportunity knocks! Br Dent J 186: 3-6.

24. Beattie A (1991) Knowledge and control in health promotion: a test case for social policy and social theory. In: Gabe J, Calnan M, Bury M (eds) The sociology of the health service. Routledge, London. 